\title{
Microscopic description of quantum Lorentz gas and extension of the Boltzmann equation to entire space-time scale
}

\author{
K. Hashimoto, ${ }^{1, *}$ K. Kanki, ${ }^{2}$ S. Tanaka, ${ }^{2}$ and T. Petrosky ${ }^{3, \dagger}$ \\ ${ }^{1}$ Graduate School of Interdisciplinary Research, University of Yamanashi, Kofu 400-8511, Japan \\ ${ }^{2}$ Department of Physical Science, Osaka Prefecture University, Sakai 599-8531, Japan \\ ${ }^{3}$ Institute of Industrial Science, The University of Tokyo, Tokyo 153-8505, Japan
}

(Received 19 November 2015; published 23 February 2016)

\begin{abstract}
Irreversible processes of weakly coupled one-dimensional quantum perfect Lorentz gas are studied on the basis of the fundamental laws of physics in terms of the complex spectral analysis associated with the resonance state of the Liouville-von Neumann operator. Without any phenomenological operations, such as a coarse-graining of space-time, or a truncation of the higher order correlation, we obtained irreversible processes in a purely dynamical basis in all space and time scale including the microscopic atomic interaction range that is much smaller than the mean-free length. Based on this solution, a limitation of the usual phenomenological Boltzmann equation, as well as an extension of the Boltzmann equation to entire space-time scale, is discussed.
\end{abstract}

DOI: 10.1103/PhysRevE.93.022132

\section{INTRODUCTION}

The world surrounding us is irreversible, and there is an arrow of time oriented toward our future that breaks time-symmetry. Nevertheless, the fundamental equations of motion in a microscopic level are time-symmetric. To resolve this apparent contradiction is a longstanding problem in dynamics and statistical mechanics. There are several different formalisms on this problem, which include the generalized master equation [1-6] or the Bogoliubov-Born-GreenKirkwood-Yvon hierarchy [7-9]. Over these formalisms, the phenomenological approximations, such as the molecular chaos and the coarse-graining of space-time, are invoked to derive the irreversible kinetic equations [10-12]. Indeed, many authors of textbooks on statistical mechanics explain that the irreversibility is not a fundamental property of dynamics, and it comes from our approximation of smearing out of detailed microscopic information that cannot be controlled due to our limitations as human beings (see, e.g., [13]).

With Ilya Prigogine, one of the authors (T.P.) has formulated the irreversible dynamics through the reversible microscopic dynamics without relying upon such phenomenological arguments [14-16]. In this formulation, we consider the spectral representation of the Liouville-von Neumann operator (Liouvillian) for systems in which irreversibility is expected such as the case of the thermodynamic limit where intensive variables and extensive variables exist. This formulation is an extension of the well-known Brillouin-Wigner-Feshbach formalism [17-19] of the eigenvalue problem of the Hamiltonian in quantum systems to the eigenvalue problem of the Liouvillian. In this formulation, we start with a complete set of projection operators that spans the Liouville space. Then, for each subspace associated to a projection operator, we construct an effective Liouvillian that shares eigenvalues with the original Liouvillian, similarly to the case for the effective Hamiltonian in the Hamiltonian dynamics $[19,20]$.

\footnotetext{
*hashimotok@yamanashi.ac.jp

${ }^{\dagger}$ Permanent address: Center for Studies in Complex Quantum Systems, The University of Texas at Austin, TX 78712, USA.
}

In this formulation, we have a self-frequency part of the effective Liouvillian corresponding to the self-energy part of the effective Hamiltonian [16]. By the same reason in the effective Hamiltonian [19,20], the effective Liouvillian may become a non-Hermitian operator for unstable systems with resonance singularity in the self-frequency part $[14,15]$, in spite of the fact that the Liouvillian itself is a Hermitian operator. As a result, an eigenvalue of the Liouvillian becomes a complex number which is located at a resonance pole of the resolvent operator of the Liouvillian in the complex frequency space. Associated to a complex eigenvalue, we have a resonance state for the Liouvillian as in the case of the Hamiltonian. The imaginary part of the complex eigenvalue gives the decay rate of the resonance state, as well as the transport coefficients associated to the irreversible process $[16,21]$. In other words, the time-symmetry breaking indicated by the complex eigenvalue of the Liouvillian is an intrinsic dynamical property.

It is worth remarking that, in our formulation, the resonance singularity which appears in the self-frequency part introduces a randomness that ensures the phenomenological assumption of the molecular chaos [22]. This is because the scattering process of the particles described by the self-frequency part gives rise to an isotropic scattering probability independent of incident direction of a particle due to the resonance singularity. This is exactly the same reason that the spontaneous emission by an excited atom is isotropic and is independent of incident direction of light in contrast to the case of the stimulated emission [23].

In spite of the parallelism between the Hamiltonian case and the Liouvillian case, there is a fundamental difference between them. Indeed, even in the case where the unperturbed Hamiltonian does not have degeneracy, the unperturbed Liouvillian has intrinsic degeneracy because the eigenvalue of the Liouvillian is given by the difference of the eigenvalues of the Hamiltonian [16]. Therefore, even in the case the self-energy of the Hamiltonian is just a c-number, the self-frequency part of the Liouvillian becomes an operator. It is shown that the self-frequency part operator of the effective Liouvillian is what is called the collision operator studied in the kinetic theory of the non-equilibrium statistical mechanics [14-16]. 
However, it is extremely complex to show the abovementioned scenario of the irreversibility on the mathematical level, because the infinitely many degrees of freedom are mutually interacting in systems that show irreversibility. Moreover, the kinetic equation derived from the eigenvalue problem of the Liouvillian has a nonlinear structure that comes from two different origins: (i) one is the well-known nonlinearity due to the appearance of the product of the one-body distribution functions due to the many-body effect, and (ii) a new type of the nonlinearity due to the fact that the effective Liouvillian itself depends on its eigenvalue as in the case of the effective Hamiltonian. Due to these complexities, we have applied this formulation so far only to the case where drastic approximations are applicable to solve the complex eigenvalue problem of the Liouvillian, such as the cases, for example, near an equilibrium state where a linearized approximation with respect to the product of the distribution functions is applicable, or the spatial inhomogeneity has a macroscopic scale at least of the order of the mean-free length [24-26]. Since one cannot analyze the dynamics in a microscopic scale much smaller than the mean-free length by this drastic approximation, we have not yet been able to demonstrate the advantage of our microscopic approach over the phenomenological coarse-graining approach.

In this paper, we present a simple model to analyze the microscopic irreversibility in terms of the complex eigenvalue problem of the Liouvillian. The model is a weakly coupled onedimensional (1D) quantum Lorentz gas $[15,27,28]$. This model is simple enough to solve the above-mentioned nonlinear problem, but still complex enough to analyze a non-trivial irreversible process such as the diffusion process. The perfect Lorentz gas consists of one light-mass test particle and infinitely many heavy particles, where the mass of the test particle is negligible as compared with the mass of each heavy particle. Because of a huge difference of the numbers of degrees of freedom between the test particle and the heavy particles, one can neglect the time evolution of the distribution function of the heavy particles in the thermodynamic limit. As a result, the nonlinearity associated to the origin (i) mentioned above does not exist in the Lorentz gas. Therefore, our main problem in this paper is to solve the new type of nonlinear problem (ii) of the effective Liouvillian mentioned above.

In this paper, we shall solve the nonlinear eigenvalue problem of the effective Liouvillian by representing the eigenvalue equation in terms of the complete eigenbasis of the phenomenological Boltzmann collision operator for the same system, which was studied in detail in [27]. The nonlinear eigenvalue problem of the effective Liouvillian is solved for the entire region of the space, which includes an atomic scale much smaller than the mean-free length. It is remarkable that irreversibility is present even in such a microscopic scale of the atomic level. As a result, the irreversibility associated to such a small spatial scale depends on a detailed form of the interaction in space.

The result is in contrast to the well-known phenomenological Boltzmann equation where the collision term does not depend on the detailed form of the interaction due to a spatial coarse-graining operation. Indeed, it is well known that the phenomenological Boltzmann equation is applicable only for a much larger length scale as compared with the atomic scale, where the detailed form of the spatial structure of the interaction is irrelevant. Here, we see the clear limitation of the phenomenological Boltzmann equation, and see that our approach goes much beyond the phenomenological coarsegraining approach.

The structure of this paper is organized as follows. In Sec. II, we introduce the model. In Sec. III, we give a brief summary of the general formalism of the complex spectral representation of the Liouvillian, and we discuss its application to the model. The effective Liouvillian for the system is derived there. In Sec. IV, we summarize the solution of the eigenvalue problem of the phenomenological Boltzmann collision operator. In Sec. V, we solve the nonlinear eigenvalue problem of the effective Liouvillian in terms of the complete eigenbasis of the Boltzmann collision operator. In Sec. VI, we discuss the structure of the spectrum of the Liouvillian with emphasis on its relation to the spectrum of the Boltzmann collision operator. In Sec. VII, we give concluding remarks. In Appendix A, we summarize the algebras in the Liouville space. In Appendix B, we discuss temperature dependence of the effective Liouvillian. In Appendix C, we summarize the extended pseudo-eigenstate representation introduced in [28] for use in Sec. VI.

\section{SYSTEM}

The Lorentz gas consists of one light-mass particle (the test particle) with mass $m$ and $N$ heavy particles with mass $M$. The Hamiltonian of the system is given by

$$
H=H_{0}+g V=\frac{p^{2}}{2 m}+\sum_{j=1}^{N} \frac{p_{j}^{2}}{2 M}+g \sum_{j=1}^{N} V\left(\left|x-x_{j}\right|\right),
$$

where $g$ is a dimensionless coupling constant and the interaction $V$ is assumed to be a short-range repulsive potential. In this paper, we consider a weak-coupling situation $(g \ll 1)$. Later we will take the limit $m / M \rightarrow 0$ in which the system is called the perfect Lorentz gas [29] [see Eq. (33)].

We assume that the system is enclosed in a large 1D box of volume $L$ with the periodic boundary condition. The interaction potential is expanded in the Fourier series as

$$
V\left(\left|x-x_{j}\right|\right)=\frac{1}{\Omega} \sum_{n} V_{q_{n}} e^{i q_{n}\left(x-x_{j}\right)},
$$

where $V_{q_{n}}=V_{\left|q_{n}\right|}, \Omega \equiv L / 2 \pi$, the wave number $q_{n}$ is given by $q_{n} \equiv n \Delta q$ with $\Delta q \equiv 1 / \Omega$ and $n=0, \pm 1, \pm 2, \ldots$ We assume $V_{q_{n}}$ is a continuous function at $q_{n}=0$ in the continuous limit $\Delta q \rightarrow 0$, and satisfies the condition

$$
\mathrm{O}\left(\left|q_{n}\right|^{3 / 2}\right)<\left|V_{q_{n}}\right|<\mathrm{O}\left(\left|q_{n}\right|^{1 / 2}\right)
$$

for $q_{n} \rightarrow 0$, in order to avoid a singular transport process characteristic in the 1D system [see Eq. (41) for the decay rate, and Eq. (60) for the diffusion coefficient].

In this paper, we consider the thermodynamic limit for the heavy particles $L \rightarrow \infty, N \rightarrow \infty$ with $c \equiv N / L=$ finite, where $c$ is the concentration of the heavy particles. In this limit, we have $\Delta q \rightarrow 0$ and the wave number and the momentum become continuous variables. At an appropriate stage, we shall replace a summation with an integration and a Kronecker delta $\delta^{K r}$ with a Dirac $\delta$-function as $\Omega^{-1} \sum_{q} \rightarrow \int d q$ and 
$\Omega_{\hbar} \delta^{K r}\left(P-P^{\prime}\right) \rightarrow \delta\left(P-P^{\prime}\right)$ with $\Omega_{\hbar} \equiv \Omega / \hbar$, respectively (hereafter we use a conventional notation $\sum_{q}$ for $\sum_{n}$ and drop the index $n$ in $q_{n}$ ).

Time evolution of the density matrix for the total $N+1$ particles system $\rho(t)$ obeys the Liouville-von Neumann equation

$$
i \frac{\partial}{\partial t} \rho(t)=L_{H} \rho(t)
$$

where $L_{H}$ is the Liouvillian that is defined by the commutation relation with the Hamiltonian as $L_{H} \rho \equiv[H, \rho] / \hbar=\left(L_{0}+\right.$ $\left.g L_{V}\right) \rho$, where $L_{0}$ is the unperturbed Liouvillian associated with $H_{0}$ and $g L_{V}$ is the interaction Liouvillian associated with $g V$.

In this paper, we focus our attention on the time evolution of the test particle. Hence we will trace out degrees of freedom of the heavy particles later [see Eq. (28)]. We denote the reduced density operator for the test particle as $f(t) \equiv \operatorname{Tr}_{\text {hev }}[\rho(t)]$, where $\operatorname{Tr}_{\text {hev }}$ is the partial trace over the heavy particles.

We assume that the initial condition of the system is given in the form

$$
\rho(0)=f(0) \otimes \rho_{\text {hev }}^{\text {eq }},
$$

where $\rho_{\text {hev }}^{\mathrm{eq}}$ is the Maxwell distribution of the heavy particles with temperature $T$,

$$
\rho_{\text {hev }}^{\mathrm{eq}} \equiv \prod_{j=1}^{N} \exp \left(-\frac{p_{j}^{2}}{2 M k_{B} T}\right) / \operatorname{Tr}\left[\exp \left(-\frac{p_{j}^{2}}{2 M k_{B} T}\right)\right],
$$

and $k_{B}$ is the Boltzmann constant. In the thermodynamic limit the time evolution of the density matrix associated with the heavy particles is negligible since its deviation from $\rho_{\text {hev }}^{\mathrm{eq}}$ is proportional to $1 / L$.

In order to discuss the "coordinate" and the "momentum" dependence of the distribution of the quantum particles in parallel with classical mechanics, let us introduce the Wigner distribution function, which is a quantum analog of the phasespace distribution function in classical mechanics $[5,15]$, defined by

$$
\begin{aligned}
& \rho^{W}\left(X,\left\{X_{j}\right\}, P,\left\{P_{j}\right\}, t\right) \\
& \equiv \frac{1}{\Omega^{N+1}} \sum_{k,\left\{k_{j}\right\}} \rho_{k,\left\{k_{j}\right\}}\left(P,\left\{P_{j}\right\}, t\right) e^{i\left(k X+k_{1} X_{1}+\cdots+k_{N} X_{N}\right)}
\end{aligned}
$$

with the Fourier component

$$
\begin{aligned}
& \rho_{k,\left\{k_{j}\right\}}\left(P,\left\{P_{j}\right\}, t\right) \\
& \equiv\left\langle P+\frac{\hbar k}{2},\left\{P_{j}+\frac{\hbar k_{j}}{2}\right\}|\rho(t)| P-\frac{\hbar k}{2},\left\{P_{j}-\frac{\hbar k_{j}}{2}\right\}\right) .
\end{aligned}
$$

Here each of the notations $\left\{X_{j}\right\},\left\{P_{j}\right\}$ represents a set of variables for the $N$ heavy particles. The momentum states of $N+1$ particles $\left|p,\left\{p_{j}\right\}\right\rangle$ is an eigenvector of $H_{0}$,

$$
H_{0}\left|p,\left\{p_{j}\right\}\right\rangle=\epsilon_{p,\left\{p_{j}\right\}}\left|p,\left\{p_{j}\right\}\right\rangle
$$

with $\epsilon_{p,\left\{p_{j}\right\}} \equiv p^{2} / 2 m+\sum_{j=1}^{N} p_{j}^{2} / 2 M$. The "wave number" $k$ and the "momentum" $P$ in the Fourier component of the Wigner representation are given in terms of the momenta in the matrix elements $\left\langle p,\left\{p_{j}\right\}|\rho(t)| p^{\prime},\left\{p_{j}^{\prime}\right\}\right\rangle$ as

$$
\begin{aligned}
& \hbar k=p-p^{\prime}, \quad P=\frac{1}{2}\left(p+p^{\prime}\right), \\
& \hbar k_{j}=p_{j}-p_{j}^{\prime}, \quad P_{j}=\frac{1}{2}\left(p_{j}+p_{j}^{\prime}\right) .
\end{aligned}
$$

\section{COMPLEX SPECTRAL REPRESENTATION OF THE LIOUVILLIAN}

\section{A. General formalism}

We briefly summarize the general formalism of the complex eigenvalue problem of the Liouvillian [14-16]. In the following, we use the Liouville space representation to formulate the eigenvalue problem of the Liouvillian. A brief summary of the Liouville space representation as well as the definitions of the notations are presented in Appendix A.

The eigenvalue problem of the Liouvillian is given by

$$
\begin{aligned}
\left.L_{H}\left|F_{\alpha}^{(v)}\right\rangle\right\rangle & \left.=Z_{\alpha}^{(v)}\left|F_{\alpha}^{(v)}\right\rangle\right\rangle, \\
\left\langle\left\langle\tilde{F}_{\alpha}^{(v)}\right| L_{H}\right. & =\left\langle\left\langle\tilde{F}_{\alpha}^{(v)}\right| Z_{\alpha}^{(v)},\right.
\end{aligned}
$$

where the double bra- and ket-vectors stand for vectors in the Liouville space (see Appendix A as well as [15]), the indices $\alpha$ and $v$ specify an eigenvalue [especially $v$ is associated to the spatial correlation (see [15])], and $\left.\left|F_{\alpha}^{(v)}\right\rangle\right\rangle$ and $\left\langle\left\langle\tilde{F}_{\alpha}^{(v)}\right|\right.$ are rightand left-eigenstates, respectively.

We apply the Brillouin-Wigner-Feshbach formalism $[15,16]$ with projection operators $P^{(v)}$ and $Q^{(v)}$ satisfying

$$
\begin{gathered}
P^{(v)} L_{0}=L_{0} P^{(v)}, \\
P^{(v)} P^{(\mu)}=\delta_{v, \mu} P^{(v)}, \\
\sum_{v} P^{(v)}=\hat{I}_{N+1},
\end{gathered}
$$

and

$$
P^{(v)}+Q^{(v)}=\hat{I}_{N+1},
$$

where $\hat{I}_{N+1}$ is the unit operator for the $N+1$ particles system. We have

$$
\left(P^{(v)}\right)^{2}=P^{(v)},\left(Q^{(v)}\right)^{2}=Q^{(v)}, P^{(v)} Q^{(v)}=0 .
$$

By applying these projection operators on Eq. (11a), we obtain a set of equations for $\left.P^{(v)}\left|F_{\alpha}^{(v)}\right\rangle\right\rangle$ and $\left.Q^{(v)}\left|F_{\alpha}^{(v)}\right\rangle\right\rangle$. By solving them for $\left.P^{(v)}\left|F_{\alpha}^{(v)}\right\rangle\right\rangle$, we obtains the nonlinear eigenvalue problem, i.e.,

$$
\left.\left.\Psi^{(v)}\left(Z_{\alpha}^{(v)}\right) P^{(v)}\left|F_{\alpha}^{(v)}\right\rangle\right\rangle=Z_{\alpha}^{(v)} P^{(v)}\left|F_{\alpha}^{(v)}\right\rangle\right\rangle,
$$

where

$$
\begin{aligned}
\Psi^{(v)}(z) \equiv & P^{(v)} L_{H} P^{(v)}+P^{(v)} L_{H} Q^{(v)} \\
& \times \frac{1}{z-Q^{(v)} L_{H} Q^{(v)}} Q^{(v)} L_{H} P^{(v)}
\end{aligned}
$$

is the effective Liouvillian. Here, the nonlinearity means that the effective Liouvillian itself depends on its eigenvalue. This is the nonlinearity of origin (ii) mentioned in the Introduction. The second term of the effective Liouvillian is the selffrequency part that corresponds to the well-known self-energy part in the case of the eigenvalue problem for the Hamiltonian 
(see, e.g., [20]). The effective Liouvillian is also called the collision operator and is a central object in non-equilibrium statistical mechanics [16]. The first term in Eq. (18) gives the flow term and the Vlasov term while the second term gives the collision term in the collision operator. In this sense, the eigenvalue equation of the effective Liouvillian is nonlinear.

It is well-known for an unstable quantum system with a continuous spectrum that the effective Hamiltonian becomes a non-Hermitian operator due to the resonance singularity in the self-energy part [20]. Similarly, the effective Liouvillian becomes a non-Hermitian operator in the Liouville space in the thermodynamic limit. As a result, the effective Liouvillian has eigenstates with complex eigenvalues that are called resonance states.

The eigenstates of the full Liouvillian are given by

$$
\begin{aligned}
& \left.\left.\left|F_{\alpha}^{(v)}\right\rangle\right\rangle=\left[P^{(v)}+\mathcal{C}^{(v)}\left(Z_{\alpha}^{(v)}\right)\right] P^{(v)}\left|F_{\alpha}^{(v)}\right\rangle\right\rangle, \\
& \left\langle\left\langle\tilde{F}_{\alpha}^{(v)}\right|=\left\langle\left\langle\tilde{F}_{\alpha}^{(v)}\right| P^{(v)}\left[P^{(v)}+\mathcal{D}^{(v)}\left(Z_{\alpha}^{(v)}\right)\right]\right.\right.
\end{aligned}
$$

with the creation-of-correlation operator

$$
\mathcal{C}^{(v)}(z)=\frac{1}{z-Q^{(v)} L_{H} Q^{(v)}} Q^{(v)} L_{H} P^{(v)},
$$

and the destruction-of-correlation operator

$$
\mathcal{D}^{(v)}(z)=P^{(v)} L_{H} Q^{(v)} \frac{1}{z-Q^{(v)} L_{H} Q^{(v)}},
$$

which are off-diagonal transitions between the $Q^{(v)}$ subspace and the $P^{(v)}$ subspace [15].

\section{B. Effective Liouvillian for the 1D quantum Lorentz gas}

Let us now apply the general formalism presented above to the weakly coupled 1D quantum perfect Lorentz gas. Using the Wigner basis $\left.\left|k,\left\{k_{j}\right\} ; P,\left\{P_{j}\right\}\right\rangle\right\rangle$ in Eq. (A15), the Fourier component of the Wigner distribution function (8) is represented by

$$
\rho_{k,\left\{k_{j}\right\}}\left(P,\left\{P_{j}\right\}, t\right)=\left\langle\left\langle k,\left\{k_{j}\right\} ; P,\left\{P_{j}\right\} \mid \rho(t)\right\rangle\right\rangle .
$$

In order to apply the Brilloun-Wigner-Feshbach formalism, we define the projection operators as

$$
\left.P^{(k)} \equiv \sum_{\mathbf{P}}\left|k,\left\{0_{j}\right\} ; \mathbf{P}\right\rangle\right\rangle\left\langle k,\left\{0_{j}\right\} ; \mathbf{P}\right|,
$$

and

$$
\left.Q^{(k)}=1-P^{(k)}=\sum_{\tilde{\mathbf{k}} \neq\left(k,\left\{0_{j}\right\}\right)} \frac{1}{\Omega_{\hbar}^{N+1}} \sum_{\tilde{\mathbf{P}}}|\tilde{\mathbf{k}} ; \tilde{\mathbf{P}}\rangle\right\rangle\langle\tilde{\mathbf{k}} ; \tilde{\mathbf{P}}|,
$$

where the bold characters like $\mathbf{P}$ are abbreviated notations for $N+1$ variables $\mathbf{P}=\left\{P, P_{1}, \ldots, P_{N}\right\}$, and the notation $\left\{0_{j}\right\}$ indicates that all wave numbers associated to the heavy particles are zero. For the projection operator $P^{(k)}$, we have

$$
\left.\left.P^{(k)} L_{0} P^{(k)}\left|k,\left\{0_{j}\right\} ; \mathbf{P}\right\rangle\right\rangle=\frac{k P}{m}\left|k,\left\{0_{j}\right\} ; \mathbf{P}\right\rangle\right\rangle,
$$

and

$$
g P^{(k)} L_{V} P^{(k)}=0,
$$

because of $V_{0}=0$ due to the assumption (3).

In the weak coupling situation, we approximate the effective Liouvillian (18) up to the second order in $g$ as

$$
\Psi_{2}^{(k)}(z)=P^{(k)} L_{0} P^{(k)}+g^{2} P^{(k)} L_{V} Q^{(k)} \frac{1}{z-L_{0}} Q^{(k)} L_{V} P^{(k)} .
$$

By using Eq. (24) and the Wigner representation of $g L_{V}$ given by Eq. (A18), we have

$$
\begin{aligned}
& \left\langle\left\langle k,\left\{0_{j}\right\} ; \mathbf{P}\left|\Psi_{2}^{(k)}(z)\right| k,\left\{0_{j}\right\} ; \mathbf{P}^{\prime}\right\rangle\right\rangle \\
& =\left[\frac{k P}{m}-\frac{1}{\Omega^{2}} \sum_{j=1}^{N} \sum_{q} \frac{g^{2}\left|V_{q}\right|^{2}}{\hbar^{2}}\left(\hat{\eta}_{P}^{\frac{\hbar}{2} q} \hat{\eta}_{P_{j}}^{-\frac{\hbar}{2} q}-\hat{\eta}_{P}^{-\frac{\hbar}{2} q} \hat{\eta}_{P_{j}}^{\frac{\hbar}{2} q}\right)\right. \\
& \left.\times \frac{1}{z-(k-q) P / m-q P_{j} / M}\left(\hat{\eta}_{P}^{\frac{\hbar}{2} q} \hat{\eta}_{P_{j}}^{-\frac{\hbar}{2} q}-\hat{\eta}_{P}^{-\frac{\hbar}{2} q} \hat{\eta}_{P_{j}}^{\frac{\hbar}{2} q}\right)\right] \\
& \quad \times \delta^{K r}\left(\mathbf{P}-\mathbf{P}^{\prime}\right),
\end{aligned}
$$

where $\delta^{K r}\left(\mathbf{P}-\mathbf{P}^{\prime}\right) \equiv \delta^{K r}\left(P-P^{\prime}\right) \prod_{j=1}^{N} \delta^{K r}\left(P_{j}-P_{j}^{\prime}\right)$, the displacement operators $\hat{\eta}_{P}^{p}, \hat{\eta}_{P_{j}}^{p}$ are defined by Eq. (A19), and we have not yet taken the limit of the perfect Lorentz gas $(m / M \rightarrow 0)$.

We focus our attention on the test particle. We define the reduced effective Liouvillian for the test particle as

$$
\bar{\Psi}_{2}^{(k)}(z) \equiv \operatorname{Tr}_{\text {hev }}\left[\Psi_{2}^{(k)}(z) \rho_{\text {hev }}^{\mathrm{eq}}\right]
$$

where we put a bar on the notation to distinguish it from the effective Liouvillian for the whole system (26). We now present an expression of its matrix element,

$$
\begin{aligned}
& \left\langle\left\langle k ; P\left|\bar{\Psi}_{2}^{(k)}(z)\right| k ; P^{\prime}\right\rangle\right\rangle \\
& \quad=\left\langle\left\langle k ; P \mid\left[\sum_{\left\{P_{j}\right\}} \frac{1}{\Omega_{\hbar}^{N}}\left\langle\left\langle\left\{0_{j}\right\} ;\left\{P_{j}\right\}\left|\Psi_{2}^{(k)}(z)\right| \rho_{\text {hev }}^{\mathrm{eq}}\right\rangle\right]\left|k ; P^{\prime}\right\rangle\right\rangle,\right.\right.
\end{aligned}
$$

where $|k ; P\rangle\rangle$ and $\left.\left|\left\{0_{j}\right\} ;\left\{P_{j}\right\}\right\rangle\right\rangle$ are reduced states for the test particle and heavy particles, respectively. Because we are interested in the thermodynamic limit with $L \rightarrow \infty$, we will replace $\Omega_{\hbar}^{-1} \sum_{P_{j}}$ by $\int_{-\infty}^{\infty} d P_{j}$. Nevertheless, keeping the summation sign for the wave vector $q$, as a convention, we obtain the following expression with the aid of Eqs. (6) and (27):

$$
\begin{aligned}
\left\langle\left\langle k ; P\left|\bar{\Psi}_{2}^{(k)}(z)\right| k ; P^{\prime}\right\rangle\right\rangle= & {\left[\frac{k P}{m}-g^{2} \frac{1}{\Omega^{2}} \sum_{j=1}^{N} \int_{-\infty}^{\infty} d P_{j} \sum_{q \neq 0} \frac{\left|V_{q}\right|^{2}}{\hbar^{2}}\left(\hat{\eta}_{P}^{\frac{\hbar}{2} q} \hat{\eta}_{P_{j}}^{-\frac{\hbar}{2} q}-\hat{\eta}_{P}^{-\frac{\hbar}{2} q} \hat{\eta}_{P_{j}}^{\frac{\hbar}{2} q}\right)\right.} \\
& \left.\times \frac{1}{z-(k-q) P / m-l P_{j} / M}\left(\hat{\eta}_{P}^{\frac{\hbar}{2} q} \hat{\eta}_{P_{j}}^{-\frac{\hbar}{2} q}-\hat{\eta}_{P}^{-\frac{\hbar}{2} q} \hat{\eta}_{P_{j}}^{\frac{\hbar}{2} q}\right) \rho_{\mathrm{hev}}^{\mathrm{eq}}\left(P_{j}\right)\right] \delta^{K r}\left(P-P^{\prime}\right),
\end{aligned}
$$


where $\rho_{\text {hev }}^{\mathrm{eq}}\left(P_{j}\right)$ is the Maxwell distribution for the heavy particle $j$ with temperature $T$,

$$
\rho_{\mathrm{hev}}^{\mathrm{eq}}\left(P_{j}\right) \equiv\left(\frac{1}{2 \pi M k_{B} T}\right)^{1 / 2} \exp \left(-\frac{P_{j}^{2}}{2 M k_{B} T}\right) .
$$

Since the mass of the test particle $m$ is much smaller than the mass of a heavy particle $M$, we expand the propagator in Eq. (30) as a power series of the ratio $m / M$. Then we have

$$
\left\langle\left\langle k ; P\left|\bar{\Psi}_{2}^{(k)}(z)\right| k ; P^{\prime}\right\rangle\right\rangle=\left\langle\left\langle k ; P\left|\psi^{(k)}(z)\right| k ; P^{\prime}\right\rangle\right\rangle+O\left(\frac{m}{M}\right),
$$

where

$$
\begin{aligned}
\langle k ; & \left.\left.P\left|\psi^{(k)}(z)\right| k ; P^{\prime}\right\rangle\right\rangle \\
= & {\left[\frac{k P}{m}-\frac{2 \pi g^{2} c}{\hbar^{2}} \frac{1}{\Omega} \sum_{q \neq 0}\left|V_{q}\right|^{2} \partial_{P}^{\hbar q / 2} \frac{1}{z-(k-q) P / m} \partial_{P}^{\hbar q / 2}\right] } \\
& \times \delta^{K r}\left(P-P^{\prime}\right)
\end{aligned}
$$

is the effective Liouvillian for the perfect Lorentz gas $(m / M \rightarrow 0)$. Here $\partial_{P}^{\hbar q / 2}$ is defined by

$$
\partial_{P}^{\hbar q / 2} \equiv \hat{\eta}_{P}^{\hbar q / 2}-\hat{\eta}_{P}^{-\hbar q / 2} .
$$

Note that there is no temperature dependent term in Eq. (33). Temperature dependence comes from the correction term to Eq. (33) starting with the first order in $(m / M)$ (see Appendix B).

For the reduced effective Liouvillian, we write the eigenvalue problem as

$$
\begin{aligned}
\left.\psi^{(k)}\left(z_{\alpha}^{(k)}\right)\left|u_{\alpha}^{(k)}\right\rangle\right\rangle & \left.=z_{\alpha}^{(k)}\left|u_{\alpha}^{(k)}\right\rangle\right\rangle, \\
\left\langle\left\langle\tilde{v}_{\alpha}^{(k)}\right| \psi^{(k)}\left(z_{\alpha}^{(k)}\right)\right. & =z_{\alpha}^{(k)} \mid\left\langle\tilde{v}_{\alpha}^{(k)}\right| .
\end{aligned}
$$

We note that $z_{\alpha}^{(k)}=Z_{\alpha}^{(k)}$ for our Lorentz gas, because the heavy particles are in an eigenstate with zero eigenvalue, i.e., they remain in thermal equilibrium.

The effective Liouvillian in Eq. (35) depends on its eigenvalue. In this sense, the eigenvalue equation is still nonlinear. Our main goal is to construct the solution of this nonlinear eigenvalue problem.

\section{THE NONLINEAR EIGENVALUE PROBLEM OF THE EFFECTIVE LIOUVILLIAN}

In this section, we solve the nonlinear eigenvalue problem of the effective Liouvillian. In order to solve the eigenvalue problem, we need to give a specific representation to the eigenvalue equations (35). For the convenience of the following discussion, here we choose the eigenbasis of the phenomenological Boltzmann collision operator for the system as a complete basis set to represent them.

The phenomenological Boltzmann collision operator is defined in the thermodynamic limit by

$$
\psi_{B}^{(k)}=P^{(k)} L_{0} P^{(k)}+g^{2} \delta \psi_{B}^{(k)}
$$

with the flow term

$$
\left\langle\left\langle k ; P\left|P^{(k)} L_{0} P^{(k)}\right| k ; P^{\prime}\right\rangle\right\rangle=\frac{k P}{m} \delta^{K r}\left(P-P^{\prime}\right),
$$

and the collision term

$$
\begin{aligned}
\langle k k ; & \left.\left.P\left|g^{2} \delta \psi_{B}^{(k)}\right| k ; P^{\prime}\right\rangle\right\rangle \\
\equiv & \left\langle\left\langle 0 ; P\left|\psi^{(0)}(+i \epsilon)\right| 0 ; P^{\prime}\right\rangle\right\rangle \\
= & -g^{2} \frac{2 \pi c}{\hbar^{2}} \frac{1}{\Omega} \sum_{q \neq 0}\left|V_{q}\right|^{2} \partial_{P}^{\hbar q / 2} \frac{1}{+i \epsilon+q P / m} \partial_{P}^{\hbar q / 2} \\
& \times \delta^{K r}\left(P-P^{\prime}\right) .
\end{aligned}
$$

Here $\epsilon$ is a positive infinitesimal satisfying $\epsilon \gg L^{-1}$ in the thermodynamic limit with $L \rightarrow \infty$.

We note that in spite of the fact that $\psi_{B}^{(k)}$ is in the $P^{(k)}$ subspace, which is orthogonal to the $P^{(0)}$ subspace, the collision term is independent of $k$ and its matrix element has the same form as $\psi_{B}^{(0)}$, which is associated with the spatially homogeneous component of the distribution function. In this sense, the Boltzmann collision operator (36) is a "coarse-grained object" in space. Thus it does not contain information of microscopic structure of the interaction.

In the article [27], the eigenvalue problem of the collision operator (38),

$$
\begin{aligned}
\left.\psi_{B}^{(k)}\left|\phi_{\alpha}^{(k)}\right\rangle\right\rangle & \left.=z_{\alpha}^{(k) B}\left|\phi_{\alpha}^{(k)}\right\rangle\right\rangle, \\
\left\langle\left\langle\tilde{\phi}_{\alpha}^{(k)}\right| \phi_{B}^{(k)}\right. & =z_{\alpha}^{(k) B}\left\langle\left\langle\tilde{\phi}_{\alpha}^{(k)}\right|,\right.
\end{aligned}
$$

has been solved. Here we summarize its solution. The eigenvalues are give by

$$
z_{ \pm ; P_{0}}^{(k) B}=-i \frac{g^{2} \gamma_{P_{0}}}{2} \pm \frac{P_{0}}{m}\left(k^{2}-k_{P_{0}}^{2}\right)^{1 / 2},
$$

where

$$
g^{2} \gamma_{P_{0}} \equiv-i g^{2} \frac{8 \pi^{2} m c}{\hbar^{2} P_{0}}\left|V_{\frac{2 P_{0}}{\hbar}}\right|^{2}
$$

is the momentum relaxation rate, and

$$
k_{P_{0}} \equiv \frac{g^{2} \gamma_{P_{0}}}{2 P_{0} / m}=l_{P_{0}}^{-1}
$$

is a wave number equals to the inverse of the mean-free length of the test particle with a momentum $P_{0}$, denoted by $l_{P_{0}}$. Corresponding eigenvectors are

$$
\begin{aligned}
& \left|\phi_{ \pm ; P_{0}}^{(k)}\right\rangle=\left|\alpha_{ \pm ; P_{0}}^{(k)}\right\rangle+\left|\beta_{ \pm ; P_{0}}^{(k)}\right\rangle, \\
& \left\langle\left\langle\tilde{\phi}_{ \pm ; P_{0}}^{(k)}\right|=\left\langle\left\langle\tilde{\alpha}_{ \pm ; P_{0}}^{(k)}\right|+\left\langle\left\langle\tilde{\beta}_{ \pm ; P_{0}}^{(k)}\right|,\right.\right.\right.
\end{aligned}
$$

where

$$
\begin{aligned}
& \left.\left|\alpha_{ \pm ; P_{0}}^{(k)}\right\rangle\left|\equiv \alpha_{ \pm ; P_{0}}^{(k)}\right| k ; P_{0}\right\rangle, \\
& \left.\left|\beta_{ \pm ; P_{0}}^{(k)}\right\rangle \equiv \beta_{ \pm ; P_{0}}^{(k)}\left|k ;-P_{0}\right\rangle\right\rangle, \\
& \left\langle\left|\tilde{\alpha}_{ \pm ; P_{0}}^{(k)}\right| \equiv \alpha_{ \pm ; P_{0}}^{(k)}\left\langle k ; P_{0}\right|,\right. \\
& \left\langle\langle \tilde { \beta } _ { \pm ; P _ { 0 } } ^ { ( k ) } | \equiv \beta _ { \pm ; P _ { 0 } } ^ { ( k ) } \left\langle\left\langle k ;-P_{0}\right|\right.\right.
\end{aligned}
$$


with

$$
\begin{aligned}
& \alpha_{ \pm ; P_{0}}^{(k)} \equiv\left[\frac{1}{2} \pm \frac{k}{2\left(k^{2}-k_{P_{0}}^{2}\right)^{1 / 2}}\right]^{1 / 2}, \\
& \beta_{ \pm ; P_{0}}^{(k)} \equiv\left[\frac{1}{2} \mp \frac{k}{2\left(k^{2}-k_{P_{0}}^{2}\right)^{1 / 2}}\right]^{1 / 2} .
\end{aligned}
$$

Since we use the eigenstates of $\psi_{B}^{(k)}$ as the basis set, it is convenient to rewrite the effective Liouvillian $\psi^{(k)}\left(z_{\alpha}^{(k)}\right)$ as follows:

$$
\psi^{(k)}\left(z_{\alpha}^{(k)}\right)=\psi_{B}^{(k)}+\lambda \Delta \psi^{(k)}\left(z_{\alpha}^{(k)}\right)
$$

with

$$
\lambda \triangle \psi^{(k)}\left(z_{\alpha}^{(k)}\right) \equiv \psi^{(k)}\left(z_{\alpha}^{(k)}\right)-\psi_{B}^{(k)},
$$

which is the difference of the effective Liouvillian and the Boltzmann collision operator We introduced a dimensionless parameter $\lambda$ for a convenience of the following discussion. From Eqs. (33) and (36), we have

$$
\lambda \triangle \psi^{(k)}\left(z_{\alpha}^{(k)}\right)=O\left(g^{2}\right) .
$$

We see that the first term in the right-hand side of Eq. (46) is diagonalized in the representation with the eigenbasis of $\psi_{B}^{(k)}$, and the order of its second term is $g^{2}$. This motivates us to solve the eigenvalue problem of Eq. (46) in terms of the standard stationary perturbation theory (see, e.g., [30]), where we regard $\psi_{B}^{(k)}$ as the unperturbed part and $\lambda \Delta \psi^{(k)}\left(z_{\alpha}^{(k)}\right)$ as the perturbation.

We expand the eigenvalues and the eigenvectors as

$$
\begin{aligned}
z_{ \pm ; P_{0}}^{(k)} & =z_{ \pm ; P_{0}}^{(k) B}+\lambda z_{ \pm ; P_{0}}^{(k) \mathrm{I}}+\lambda^{2} z_{ \pm ; P_{0}}^{(k) I I}+\cdots, \\
\left|u_{ \pm ; P_{0}}^{(k)}\right\rangle & \left.\left.=\left|\phi_{ \pm ; P_{0}}^{(k)}\right\rangle\right\rangle+\lambda\left|u_{ \pm ; P_{0}}^{(k) \mathrm{I}}\right\rangle\left|+\lambda^{2}\right| u_{ \pm ; P_{0}}^{(k) I I}\right\rangle+\cdots, \\
\left\langle\left\langle\tilde{v}_{ \pm ; P_{0}}^{(k)}\right|\right. & =\left\langle\left\langle\tilde{\phi}_{ \pm ; P_{0}}^{(k)}\right|+\lambda\left\langle\left\langle\tilde{v}_{ \pm ; P_{0}}^{(k) \mathrm{I}}\right|+\lambda^{2}\left\langle\left\langle\tilde{v}_{ \pm ; P_{0}}^{(k) I I}\right|+\cdots .\right.\right.\right.
\end{aligned}
$$

Substituting Eqs. (46), (49a), and (49b) into Eq. (35a), we have the expression of the first order correction term of the eigenvalues as

$$
\lambda z_{ \pm ; P_{0}}^{(k) I}=\left\langle\left\langle\tilde{\phi}_{ \pm ; P_{0}}^{(k)}\left|\lambda \triangle \psi^{(k)}\left(z_{ \pm ; P_{0}}^{(k)}\right)\right| \phi_{ \pm ; P_{0}}^{(k)}\right\rangle .\right.
$$

We substitute Eq. (50) to its right-hand side and expand as a power series of $\lambda$. Thus we have

$$
\lambda z_{ \pm ; P_{0}}^{(k) I}=\left\langle\left\langle\tilde{\phi}_{ \pm ; P_{0}}^{(k)}\left|\lambda \Delta \psi^{(k)}\left(z_{ \pm ; P_{0}}^{(k) B}\right)\right| \phi_{ \pm ; P_{0}}^{(k)}\right\rangle\right\rangle+O\left(g^{4}\right) .
$$

Hence, from Eq. (49a), we have

$$
z_{ \pm ; P_{0}}^{(k)}=z_{ \pm ; P_{0}}^{(k) B}+\left\langle\left\langle\tilde{\phi}_{ \pm ; P_{0}}^{(k)}\left|\lambda \triangle \psi^{(k)}\left(z_{ \pm ; P_{0}}^{(k) B}\right)\right| \phi_{ \pm ; P_{0}}^{(k)}\right\rangle\right\rangle+O\left(g^{4}\right) .
$$

By using Eqs. (43) to (52) and taking the thermodynamic limit, we obtain

$$
z_{ \pm ; P_{0}}^{(k)}=z_{ \pm ; P_{0}}^{(k) B}+\triangle z_{ \pm ; P_{0}}^{(k)[\alpha, \alpha]}+\triangle z_{ \pm ; P_{0}}^{(k)[\beta, \beta]}+O\left(g^{4}\right)
$$

with

$$
\triangle z_{ \pm ; P_{0}}^{\left.(k), \xi^{\prime}\right]} \equiv\left\langle\left\langle\tilde{\xi}_{ \pm ; P_{0}}^{(k)}\left|\lambda \Delta \psi^{(k)}\left(z_{ \pm ; P_{0}}^{(k) B}\right)\right| \xi_{ \pm ; P_{0}}^{\prime(k)}\right\rangle\right\rangle
$$

where $\xi, \xi^{\prime}=\alpha$ or $\beta$ in Eq. (43). In Eq. (53), cross terms $\triangle z_{ \pm ; P_{0}}^{(k)[\alpha, \beta]}$ and $\Delta z_{ \pm ; P_{0}}^{(k)[\beta, \alpha]}$ have vanished in the thermodynamic limit since they are proportional to $\Omega^{-1}=2 \pi / L$. This is because the summation over $q$ in the collision terms (33) and (38) can be taken for the cross terms since they include Kronecker's deltas $\delta^{K r}\left(\hbar q \pm 2 P_{0}\right)$, and thus the volume factor $\Omega^{-1}$ in front of the summation is left alone.

Explicit expressions of $\Delta z_{ \pm ; P_{0}}^{(k)[\alpha]}$ and $\triangle z_{ \pm ; P_{0}}^{(k)[\beta, \beta]}$ are

$$
\begin{aligned}
\triangle z_{ \pm ; P_{0}}^{(k) \alpha \alpha]}= & g^{2} \frac{\pi c}{\hbar^{2}}\left[1 \pm \frac{k}{\left(k^{2}-k_{P_{0}}^{2}\right)^{1 / 2}}\right] \int_{-\infty}^{\infty} d q\left|V_{q}\right|^{2} \\
& \times\left\{\frac{1}{\left[z_{ \pm ; P_{0}}^{(k) B}-(k-q)\left(P_{0}+\hbar q / 2\right) / m\right]_{+}}\right. \\
& \left.+\frac{1}{\left[z_{ \pm ; P_{0}}^{(k) B}-(k-q)\left(P_{0}-\hbar q / 2\right) / m\right]_{+}}\right\} \\
& +i \frac{g^{2} \gamma_{P_{0}}}{4}
\end{aligned}
$$

and

$$
\begin{aligned}
\triangle z_{ \pm ; P_{0}}^{(k)[\beta, \beta]}= & g^{2} \frac{\pi c}{\hbar^{2}}\left[1 \mp \frac{k}{\left(k^{2}-k_{P_{0}}^{2}\right)^{1 / 2}}\right] \int_{-\infty}^{\infty} d q\left|V_{q}\right|^{2} \\
& \times\left\{\frac{1}{\left[z_{ \pm ; P_{0}}^{(k) B}+(k-q)\left(P_{0}+\hbar q / 2\right) / m\right]_{+}}\right. \\
& \left.+\frac{1}{\left[z_{ \pm ; P_{0}}^{(k) B}+(k-q)\left(P_{0}-\hbar q / 2\right) / m\right]_{+}}\right\} \\
& +i \frac{g^{2} \gamma_{P_{0}}}{4},
\end{aligned}
$$

where we use the integration sign on $q$ since we have taken the thermodynamic limit with $L \rightarrow \infty$, and the notation $[\cdots]_{+}$ in the denominators means that the integration is evaluated in the Riemann sheet analytically continued from the upper half plane of the complex $z$ plane $[14,15]$.

Note that the expressions in Eq. (55) have singularities at $k= \pm k_{P_{0}}$. The singularities are coming from the Jordan block structure of the Boltzmann collision operator for the 1D quantum perfect Lorentz gas [28]. Therefore, the perturbation analysis with the eigenvectors performed in this section breaks down in a domain near the singular points $k= \pm k_{P_{0}}$. For this case, we have shown that there is a divergencefree representation of the collision operator by introducing extended pseudo-eigenstates in the vicinity of the singular points [28]. Using this representation, we shall discuss this domain separately in the next section.

\section{STRUCTURE OF THE SPECTRUM IN VARIOUS SPATIAL SCALES}

In this section, we discuss the structure of the spectrum of the Liouvillian in the wave number $k$ space.

Let us first summarize the results presented in this section. We show $k$ dependence of the spectrum of the Liouvillian in Fig. 1. In these figures, we show only the domain $k \geqslant 0$. The spectrum in the domain $k<0$ are symmetric with respect to the axis with $k=0$. Figure 1(a) is the imaginary part of the spectrum and Fig. 1(b) is the real part, respectively. In 

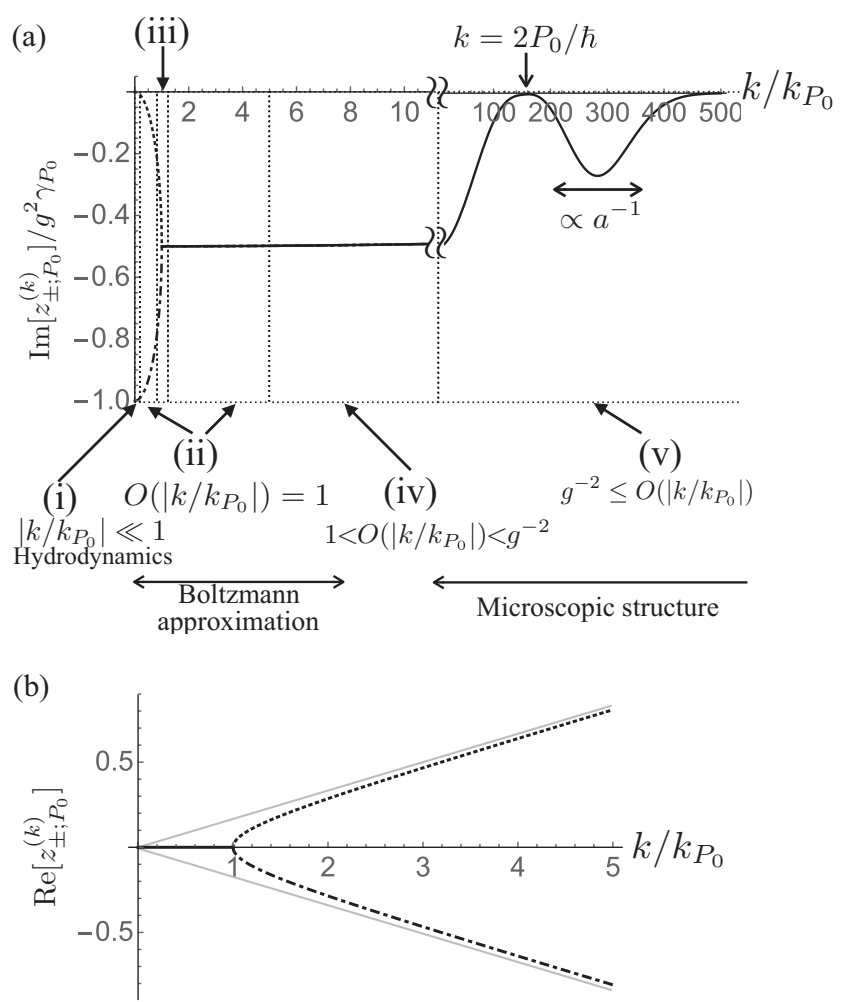

FIG. 1. Both imaginary part and real part of the spectrum of the Liouvillian is presented with respect to the wave number $k$. The figure (a) is the imaginary part and (b) is the real part. In each figure, the dotted lines represent $z_{+; P_{0}}^{(k)}$ and the dot-dashed lines represent $z_{-; P_{0}}^{(k)}$, respectively. The black lines represent that these two lines are overlapping. The gray lines in (b) are eigenvalues for a free test particle $\pm k P_{0} / m$. The five domains enclosed by the dotted squares are introduced to discuss the structure of the spectrum. The definitions of the domains are summarized in Table I.

each figure, the thick dotted lines represent $z_{+; P_{0}}^{(k)}$ and the thick dot-dashed lines represent $z_{-; P_{0}}^{(k)}$, respectively. The black lines represent that these two lines are overlapping. The gray lines in Fig. 1(b) represent eigenvalues of the Liouvillian for the free test particle $\pm k P_{0} / m$. Here we chose the following function as a interaction between particles:

$$
V_{k}=v k^{2} \exp \left[-(a k)^{2}\right],
$$

where the factor $k^{2}$ in front of the exponential is introduced for satisfying the condition (3), $v$ is the strength of the interaction and $a$ is the range of the interaction in $X$-space.

In Fig. 1(a), we decompose the region into five domains with respect to the value of $k$. These are

(i) $O\left(\left|\frac{k}{k_{P_{0}}}\right|\right) \leqslant g$,

(ii) $O\left(\left|\frac{k}{k_{P_{0}}}\right|\right)=1$ with $O\left(|| \frac{k}{k_{P_{0}}}|-1|\right)>g^{2}$,

(iii) $O\left(|| \frac{k}{k_{P_{0}}}|-1|\right) \leqslant g^{2}$,

(iv) $1<O\left(\left|\frac{k}{k_{P_{0}}}\right|\right)<g^{-2}$ or $O\left(\left|\frac{k}{k_{P_{0}}}\right|\right)=g^{-\zeta}$

with $0<\zeta<2$.

(v) $O\left(\left|\frac{k}{k_{P_{0}}}\right|\right) \geqslant g^{-2}$.
TABLE I. Key features of the spectrum.

\begin{tabular}{ll}
\hline \hline Domain & \multicolumn{1}{c}{ Key features } \\
\hline & $z_{ \pm ; P_{0}}^{(k)}=z_{ \pm ; P_{0}}^{(k) B}+O\left(g^{4}\right)$. \\
(i) & The Burnett expansion is possible. \\
& Its dominant term gives diffusion coefficient. \\
(ii) & $z_{ \pm ; P_{0}}^{(k)}=z_{ \pm ; P_{0}}^{(k) B}+O\left(g^{4}\right)$. \\
(iii) & $z_{ \pm ; P_{0}}^{(k)}=z_{ \pm ; P_{0}}^{(k) B}+O\left(g^{4}\right)$. \\
& $z_{ \pm ; P_{0}}^{(k)}=z_{ \pm ; P_{0}}^{(k) B}+O\left(g^{4-\zeta}\right)$ with $0<\zeta<2$. \\
& The Boltzmann approximation is getting bad \\
& as the order of $k$ approachs to $\left|k / k_{P_{0}}\right|=O\left(g^{-2}\right)$. \\
& The Boltzmann approximation breaks down. \\
& The spectrum has a structure reflecting \\
the form of the interaction potential. \\
(v) $\quad$ The eigenstates still have non-zero decvay rate \\
even in the spatial scale of interaction range.
\end{tabular}

The expression of the spectrum of the Liouvillian are given by Eq. (53) except for the domain (iii). The key features of the spectrum presented in the following discussion is summarized in Table I. Now we discuss the features of the spectrum in each domain: (i), (ii), (iv), (v), and finally (iii).

\section{Domain (i)}

In domain (i), the order of the wave number $k$ satisfies $O(k) \leqslant g k_{P_{0}}$. Since $k_{P_{0}}$ is a $g^{2}$ order quantity, the order of the wave number $k$ is bounded by $g^{3}$. Thus we expand $\triangle z_{ \pm ; P_{0}}^{(k)[\alpha, \alpha]}$ and $\triangle z_{ \pm ; P_{0}}^{(k)[\beta, \beta]}$ as a power series of the $g^{3}$. Then we see that the first terms in both Eqs. (55a) and (55b) give $-i g^{2} \gamma_{P_{0}} / 4+$ $O\left(g^{5}\right)$. Thus we have

$$
O\left(\triangle z_{ \pm ; P_{0}}^{(k)[\alpha, \alpha]}\right)=O\left(\triangle z_{ \pm ; P_{0}}^{(k)[\beta, \beta]}\right)=g^{5} .
$$

Combining it with Eq. (53), we see that the eigenvalues in this domain reduce to the eigenvalues of the Boltzmann collision operator (40),

$$
\begin{aligned}
z_{ \pm ; P_{0}}^{(k)} & =z_{ \pm ; P_{0}}^{(k) B}+O\left(g^{4}\right) \\
& =-i g^{2} \frac{\gamma_{P_{0}}}{2} \pm\left(k^{2}-k_{P_{0}}^{2}\right)^{1 / 2}+O\left(g^{4}\right)
\end{aligned}
$$

where the $g^{4}$ order leading term of the correction comes from the fourth term in Eq. (53).

Since $k / k_{P_{0}}$ is a small quantity, we can expand the second term of Eq. (58) into a series called as the Burnett expansion (see, e.g., [31]),

$$
z_{+; P}^{(k)}=-i \frac{g^{2} \gamma_{P}}{4}\left(\frac{k}{k_{P}}\right)^{2}-i \frac{g^{2} \gamma_{P}}{16}\left(\frac{k}{k_{P}}\right)^{4}+O\left(\frac{k}{k_{P}}\right)^{6}
$$

and

$$
\begin{aligned}
z_{-; P}^{(k)}= & -i g^{2} \gamma_{P}+i \frac{g^{2} \gamma_{P}}{4}\left(\frac{k}{k_{P}}\right)^{2}+i \frac{g^{2} \gamma_{P}}{16}\left(\frac{k}{k_{P}}\right)^{4} \\
& +O\left(\frac{k}{k_{P}}\right)^{6}
\end{aligned}
$$


The first few terms of the expansion (59a) give transport coefficients of hydrodynamic equations. For instance, the first term, which is second order in $k$, gives the diffusion coefficient,

$$
D_{P} \equiv \frac{g^{2} \gamma_{P}}{4 k_{P}^{2}}=\frac{(P / m)^{2}}{g^{2} \gamma_{P}} .
$$

We have $D_{P} \rightarrow 0$ for $P \rightarrow 0$ [see Eq. (3)]. The higher-order terms of the expansion also give transport coefficients of the Burnett equation [31].

\section{Domain (ii)}

In domain (ii), the wave number $k$ is the same order as $k_{P_{0}}$. Since $k_{P_{0}}$ is a $g^{2}$ order quantity [see Eq. (42)], the wave number $k$ is also a $g^{2}$ order quantity. Thus we see that the first term in both Eqs. (55a) and (55b) give $-i g^{2} \gamma_{P_{0}} / 4+O\left(g^{4}\right)$. Hence we have

$$
O\left(\triangle z_{ \pm ; P_{0}}^{(k) \alpha \alpha]}\right)=O\left(\triangle z_{ \pm ; P_{0}}^{(k)[\beta, \beta]}\right)=g^{4} .
$$

By combining these results with the expression (53), we see that the eigenvalues of the Liouvillian reduces to the eigenvalues of the Boltzmann collision operator,

$$
z_{ \pm ; P_{0}}^{(k)}=z_{ \pm ; P_{0}}^{(k) B}+O\left(g^{4}\right),
$$

where the $g^{4}$ order leading term of the correction comes not only from the fourth term in Eq. (53) but also from both $\triangle z_{ \pm ; P_{0}}^{(k)[\alpha, \alpha]}$ and $\triangle z_{ \pm ; P_{0}}^{(k)[\beta, \beta]}$. Thus we see that the deviation of the eigenvalues of the Liouvillian from the eigenvalues of the Boltzmann collision operator is $g^{4}$ order in this domain. Therefore, the phenomenological Boltzmann equation is valid in the domain.

\section{Domain (iv)}

We consider the domain

$$
\left|\frac{k}{k_{P_{0}}}\right|=O\left(g^{-\zeta}\right) \quad \text { with } 0<\zeta<2 .
$$

Since $k_{P_{0}}$ is a $g^{2}$ order quantity, order of the wave number $k$ is evaluated as $g^{2-\zeta}$ with $2-\zeta>0$ in this domain. Therefore, by expanding the first term in the expressions (55) as a power series of $g^{2-\zeta}$ order quantity, we see that the first term in both Eqs. (55a) and (55b) give $-i g^{2} \gamma_{P_{0}} / 4+O\left(g^{4-\zeta}\right)$. Thus we have

$$
O\left(\triangle z_{ \pm ; P_{0}}^{(k)[\alpha, \alpha]}\right)=O\left(\triangle z_{ \pm ; P_{0}}^{(k)[\beta, \beta]}\right)=g^{4-\zeta} .
$$

In this case, the leading term in Eq. (53) is $z_{ \pm ; P_{0}}^{(k) B}$ and the correction starts from the $g^{4-\zeta}$ order term, i.e.,

$$
z_{ \pm ; P_{0}}^{(k)}=z_{ \pm ; P_{0}}^{(k) B}+O\left(g^{4-\zeta}\right) .
$$

Here we observe that the precision of the Boltzmann approximation is getting worse continuously as the order of $k / k_{P_{0}}$ approaches to $g^{-2}$. As a consequence, we see that the condition

$$
O\left(\left|\frac{k}{k_{P}}\right|\right)<g^{-2}
$$

is the upper limit of the domain where the Boltzmann approximation is applicable.

\section{Domain (v)}

In domain (v), we have $O\left(\left|k_{P_{0}} / k\right|\right) \leqslant g^{2}$. Thus we can expand the expressions in Eq. (55) as power series of $k_{P_{0}} / k$. Keeping the dominant term, we have

$$
\begin{aligned}
& \Delta z_{ \pm ; P_{0}}^{(k)[\alpha]}=\left[g^{2} \frac{2 \pi n}{\hbar^{2}} \lim _{\epsilon \rightarrow+0} \int_{-\infty}^{\infty} d q\left|V_{q}\right|^{2} \frac{1}{+i \epsilon \pm q\left(\hbar q+2 P_{0}-\hbar k\right) / 2 m}+O\left(g^{4}\right)\right]+i \frac{g^{2} \gamma_{P_{0}}}{4} \\
& \triangle z_{ \pm ; P_{0}}^{(k)[\beta]}=\left[g^{2} \frac{2 \pi n}{\hbar^{2}} \lim _{\epsilon \rightarrow+0} \int_{-\infty}^{\infty} d q\left|V_{q}\right|^{2} \frac{1}{+i \epsilon \mp q\left(\hbar q-2 P_{0}-\hbar k\right) / 2 m}+O\left(g^{4}\right)\right]+i \frac{g^{2} \gamma_{P_{0}}}{4} .
\end{aligned}
$$

Hence we obtain

$$
\begin{aligned}
z_{ \pm ; P_{0}}^{(k)}= & -i g^{2} \frac{2 \pi^{2} m n}{\hbar^{2}}\left(\frac{\mid V_{k-2 P_{0} /\left.\hbar\right|^{2}}}{\left|P_{0}-\hbar k / 2\right|}+\frac{\mid V_{k+2 P_{0} /\left.\hbar\right|^{2}}}{\left|P_{0}+\hbar k / 2\right|}\right) \pm \frac{P_{0}}{m}\left(k^{2}-k_{P_{0}}^{2}\right)^{1 / 2} \\
& \pm g^{2} \frac{2 \pi m n}{\hbar^{2}} \mathcal{P} \int_{-\infty}^{\infty} d q\left|V_{q}\right|^{2}\left[\frac{1}{q\left(\hbar q+2 P_{0}-\hbar k\right) / 2}-\frac{1}{q\left(\hbar q-2 P_{0}-\hbar k\right) / 2}\right]+O\left(g^{4}\right),
\end{aligned}
$$

where $\mathcal{P}$ stands for the principal part. We note that each term in the parenthesis in the first term does not diverge at $k= \pm 2 P_{0} / \hbar$ due to the condition (3).

From the expression in Eq. (68), one can see that the structure of the spectrum in the domain reflects the form of the interaction potential $V_{k}$. Indeed, the bumped shape around $k=2 P_{0} / \hbar$ of the imaginary part of the spectrum presented in Fig. 1(a) reflects the form of the interaction potential (56). For instance, the rising of the decay rate, given by $\left|\operatorname{Im}\left[z_{ \pm ; P_{0}}^{(k)}\right]\right|$, from $k=2 P_{0} / \hbar$ is coming from the factor $k^{2}$ in front of the exponential in Eq. (56), and the width of the "hill" structure is proportional to $a^{-1}$. In this sense, the structure of the spectrum in the domain strongly depends on explicit form of interaction potential. This is in contrast to the case of the Boltzmann collision operator $\psi_{B}^{(k)}$, where the imaginary part of the spectrum (40) is independent of $k$ for any $k \geqslant k_{P_{0}}$, which results from a degradation of microscopic information by the coarse-graining.

It is remarkable that the dissipation associated with the imaginary part of the eigenvalue of the Liouvillian does not vanish in the non-coarse-grained microscopic dynamics where the microscopic structure of the form of the interaction 
is visible. In other words, the irreversibility is a direct consequence of the microscopic dynamics, and is not a result of coarse-graining operation to the equation of motion due to our limitation of the controllability of the system with a huge degrees of freedom often stated in the literature (see, e.g., [13]).

The imaginary part of the eigenvalues asymptotically vanishes for $|k| \gg k_{P_{0}}$. This property holds for any interaction potentials satisfying the condition

$$
\left|V_{q}\right|=o\left(|q|^{1 / 2}\right)
$$

for $|q| \rightarrow \infty$. Hence, for $|k| \gg k_{P_{0}}$, the eigenvalues asymptotically approach to the eigenvalues of the free particle as

$$
z_{ \pm ; P_{0}}^{(k)} \rightarrow \pm \frac{k P_{0}}{m}
$$

\section{Domain (iii)}

Let us now consider domain (iii) where the expressions (55) has singularity. In this domain, we use the extended pseudoeigenstates [28], which are free-from divergence at $k= \pm k_{P_{0}}$, as a basis set to represent the correction term. Here we denote the new basis as $\left\{\left|\chi_{+; P_{0}}^{(k)}\right\rangle,\left|\chi_{-; P_{0}}^{\prime(k)}\right\rangle,\left\langle\left\langle\tilde{\chi}_{+; P_{0}}^{\prime(k)}\right|,\left\langle\left\langle\tilde{\chi}_{-; P_{0}}^{(k)}\right|\right\}\right.\right.$. We give a brief summary of their definition as well as their expressions in Appendix $\mathrm{C}$ for use in the following discussion.

In terms of the new basis with their expressions in Eqs. (C4) with (C5), an expression of a matrix element of the correction $\lambda \triangle \psi^{(k)}\left(z_{+; P_{0}}^{(k)}\right)$ is given by

$$
\begin{aligned}
\left\langle\left\langle\tilde{\chi}_{+; P_{0}}^{(k)}\left|\lambda \Delta \psi^{(k)}\left(z_{+; P_{0}}^{(k) B}\right)\right| \chi_{+; P_{0}}^{(k)}\right\rangle=\right. & \frac{1}{2}\left\{\left[1+\frac{\left(k^{2}-k_{P_{0}}^{2}\right)^{1 / 2}}{k}\right]\left\langle\left\langle k ; P_{0}\left|\lambda \Delta \psi^{(k)}\left(z_{\alpha}^{(k) B}\right)\right| k ; P_{0}\right\rangle\right\rangle\right. \\
& \left.+\left[1-\frac{\left(k^{2}-k_{P_{0}}^{2}\right)^{1 / 2}}{k}\right]\left\langle k ;-P_{0}\left|\lambda \Delta \psi^{(k)}\left(z_{\alpha}^{(k) B}\right)\right| k ;-P_{0}\right\rangle\right\rangle \\
& \left.-i \frac{k_{P_{0}}}{k}\left\langle\left\langle k ; P_{0}\left|\lambda \Delta \psi^{(k)}\left(z_{\alpha}^{(k) B}\right)\right| k ;-P_{0}\right\rangle-i \frac{k_{P_{0}}}{k}\left\langle k ;-P_{0}\left|\lambda \Delta \psi^{(k)}\left(z_{\alpha}^{(k) B}\right)\right| k ; P_{0}\right\rangle\right\rangle\right\} .
\end{aligned}
$$

Here we have four matrix elements of the correction in the Wigner representation. By using Eqs. (33) and (38), we have the following expressions for the diagonal elements:

$$
\begin{aligned}
& \left\langle\left\langle k ; \pm P_{0}\left|\lambda \Delta \psi^{(k)}\left(z_{+; P_{0}}^{(k)}\right)\right| k ; \pm P_{0}\right\rangle\right\rangle \\
& \quad=g^{2} \frac{2 \pi c}{\hbar^{2}} \int_{-\infty}^{\infty} d q\left|V_{q}\right|^{2}\left\{\frac{1}{\left[z_{ \pm ; P_{0}}^{(k) B} \mp(k-q)\left(P_{0}+\hbar q / 2\right) / m\right]_{+}}+\frac{1}{\left[z_{ \pm ; P_{0}}^{(k) B} \mp(k-q)\left(P_{0}-\hbar q / 2\right) / m\right]_{+}}\right\}+i \frac{g^{2} \gamma_{P_{0}}}{2} .
\end{aligned}
$$

In domain (iii), the wave number $k$ is a $g^{2}$ order quantity, since $k$ is same order with the $g^{2}$ order quantity $k_{P_{0}}$. Thus we see that the first term in Eq. (72) gives $-i g^{2} \gamma_{P_{0}} / 2+O\left(g^{4}\right)$. Hence, we have

$$
O\left(\left\langle\left\langle k ; \pm P_{0}\left|\lambda \triangle \psi^{(k)}\left(z_{+; P_{0}}^{(k)}\right)\right| k ; \pm P_{0}\right\rangle\right)=g^{4} .\right.
$$

On the other hand, for the cross terms $\left\langle\left\langle k ; \pm P_{0}\right| \lambda \triangle\right.$ $\left.\psi^{(k)}\left(z_{+; P_{0}}^{(k)}\right)\left|k ; \mp P_{0}\right\rangle\right\rangle$, we see that they are proportional to $L^{-1}$ by exactly the same reason for $\Delta z_{+; P_{0}}^{(k)[\alpha, \beta]}$ and $\Delta z_{+; P_{0}}^{(k)[\beta, \alpha]}$ in Eq. (54). As a result, in the thermodynamic limit with $L \rightarrow \infty$, we have

$$
O\left(\left\langle\left\langle\tilde{\chi}_{+; P_{0}}^{(k)}\left|\lambda \Delta \psi^{(k)}\left(z_{+; P_{0}}^{(k) B}\right)\right| \chi_{+; P_{0}}^{(k)}\right\rangle\right)=g^{4} .\right.
$$

Similarly, we have

$$
\begin{aligned}
& O\left(\left\langle\left\langle\tilde{\chi}_{+; P_{0}}^{\prime(k)}\left|\lambda \Delta \psi^{(k)}\left(z_{-; P_{0}}^{(k) B}\right)\right| \chi_{-; P_{0}}^{\prime(k)}\right\rangle\right)=g^{4},\right. \\
& O\left(\left\langle\left\langle\tilde{\chi}_{-; P_{0}}^{(k)}\left|\lambda \Delta \psi^{(k)}\left(z_{+; P_{0}}^{(k) B}\right)\right| \chi_{+; P_{0}}^{(k)}\right\rangle\right)=g^{4},\right.
\end{aligned}
$$

and

$$
O\left(\left\langle\left\langle\tilde{\chi}_{-; P_{0}}^{(k)}\left|\lambda \triangle \psi^{(k)}\left(z_{-; P_{0}}^{(k) B}\right)\right| \chi_{-; P_{0}}^{\prime(k)}\right\rangle\right)=g^{4} .\right.
$$

Since the difference between the effective Liouvillian and the Boltzmann collision operator is $g^{4}$ order in domain (iii), we can conclude that the correction term of the spectrum starts from $g^{4}$ order term, i.e.,

$$
z_{ \pm ; P_{0}}^{(k)}=z_{ \pm ; P_{0}}^{(k) B}+O\left(g^{4}\right) .
$$

\section{CONCLUDING REMARKS}

We have solved the eigenvalue problem of the Liouvillian for the weakly coupled 1D quantum perfect Lorentz gas for entire space and time scales by the complex spectral analysis. The result shows that irreversibility is a purely dynamical property coming from the structure of the Liouvillian outside the Hilbert space. It is remarkable that irreversibility holds in a microscopic atomic scale, where if we would perform non-dynamical operations in the equation of motion, such as coarse-graining procedure in space and/or time, or statistical procedure with the assumption of the molecular chaos in phenomenological approach, we would lose such microscopic information because of a degradation of the information. As we have shown in the subsection "Domain (v)" in Sec. V, as well as in Fig. 1(a), the decay rate of the resonance state in this domain explicitly depends on the shape of the Fourier component of the interaction (56) in such small atomic scale.

Moreover, we have shown a precise limitation of the phenomenological Boltzmann equation for the weakly coupled 
system. As shown in Eq. (65), it is remarkable that the phenomenological Boltzmann equation is valid up to $O\left(\left|k / k_{P}\right|\right)<$ $g^{-2}$, i.e., it is valid in extremely small length scale, much smaller than the mean-free length. Of course, because the phenomenological Boltzmann equation does not depends on a detailed form of the interaction due to the coarse-graining, this equation is not applicable to a microscopic atomic scale, which is in contrast to the purely dynamical approach with our eigenvalue problem of the Liouvillian based on the complex spectral analysis.

Because we have solved the eigenvalue problem of the Liouvillian without making any coarse-graining operations, we can analyze detailed irreversible time evolution of the system in all domains of the length scale including atomic scale. We hope to present these details elsewhere.

\section{ACKNOWLEDGMENTS}

We would like to express our sincere gratitude to Prof. H. Hayakawa for motivating us to launch this study and for his valuable comments. We also thank Prof. C. Uchiyama for her valuable comments. This work was supported by JSPS KAKENHI Grant No. 24540411.

\section{APPENDIX A: LIOUVILLE SPACE}

In this appendix, we give a brief summary of the Liouville space representation of the Hilbert space operators (see [15] for more detail).

The Liouville space is spanned by linear operators $A, B$, ...in the ordinary wave function space. We represent these linear operators by double bra-ket notations as $|A\rangle\rangle,|B\rangle\rangle, \ldots$ in order to distinguish them from the bra-ket notation in the wave function space. The inner product in the Liouville space is defined by

$$
\langle\langle B \mid A\rangle\rangle \equiv \operatorname{Tr}\left[B^{+} A\right],
$$

where $B^{+}$is the Hermitian conjugate of a linear operator $B$ in the wave function space.

In the Liouville space, we can consider a linear operator $\mathcal{T}$ acting on vectors, which is operators in the wave function space. We call it a super-operator in case we want to emphasize the difference between it from the linear operator in the wave function space. One can define a super-operator $\mathcal{T}^{\dagger}$ that is the Hermitian conjugate to $\mathcal{T}$ as

$$
\left\langle\left\langle A\left|\mathcal{T}^{\dagger}\right| B\right\rangle\right\rangle \equiv(\langle\langle B|\mathcal{T}| A\rangle\rangle)^{c . c .},
$$

where the notation c.c. means the complex conjugate. Then, one can introduce the Hermitian super-operator which satisfies

$$
\mathcal{T}^{\dagger}=\mathcal{T}
$$

and the unitary super-operator which satisfies

$$
U^{\dagger}=U^{-1}
$$

The Liouvillian is an example of the Hermitian super-operators in the Liouville space, i.e.,

$$
L_{H}^{\dagger}=L_{H},
$$

and the time evolution operator,

$$
\mathcal{U}(t)=e^{-i L_{H} t},
$$

is an example of the unitary super-operators.

We now introduce a basis set in the Liouville space for 1D quantum Lorentz gas in terms of the eigenstates of $H_{0}$ in Eq. (9). Let us introduce an abbreviated notation of the $N+1$ variables $\mathbf{p}=\left\{p, p_{1}, \ldots, p_{N}\right\}$ to avoid heavy notations. Then, we express the eigenstate and the eigenvalue of $H_{0}$ in Eq. (9) as $|\mathbf{p}\rangle$ and $\epsilon_{\mathbf{p}}$. The eigenstates of $H_{0}$ satisfy the orthonormality relation

$\left\langle\mathbf{p} \mid \mathbf{p}^{\prime}\right\rangle=\delta^{K r}\left(\mathbf{p}-\mathbf{p}^{\prime}\right) \equiv \delta^{K r}\left(p-p^{\prime}\right) \prod_{j=1}^{N} \delta^{K r}\left(p_{j}-p_{j}^{\prime}\right)$,

and the completeness relation

$$
\sum_{\mathbf{p}}|\mathbf{p}\rangle\langle\mathbf{p}|=1
$$

We define vectors in the Liouville space by

$$
\left.\left|\mathbf{p}, \mathbf{p}^{\prime}\right\rangle\right\rangle \equiv|\mathbf{p}\rangle\left\langle\mathbf{p}^{\prime}\right| \text {. }
$$

These vectors are eigenstates of $L_{0}$,

$$
\left.\left.L_{0}\left|\mathbf{p}, \mathbf{p}^{\prime}\right\rangle\right\rangle=\frac{1}{\hbar}\left(\epsilon_{\mathbf{p}}-\epsilon_{\mathbf{p}^{\prime}}\right)\left|\mathbf{p}, \mathbf{p}^{\prime}\right\rangle\right\rangle .
$$

The set of eigenstates $\left.\left\{\left|\mathbf{p}, \mathbf{p}^{\prime}\right\rangle\right\rangle\right\}$ forms a orthonormal basis in the Liouville space [see Eq. (A1)]

$$
\left\langle\left\langle\mathbf{p}, \mathbf{p}^{\prime} \mid \mathbf{p}^{\prime \prime}, \mathbf{p}^{\prime \prime \prime}\right\rangle\right\rangle=\delta^{K r}\left(\mathbf{p}-\mathbf{p}^{\prime \prime}\right) \delta^{K r}\left(\mathbf{p}^{\prime}-\mathbf{p}^{\prime \prime \prime}\right),
$$

and the completeness relation

$$
\left.\sum_{\mathbf{p}} \sum_{\mathbf{p}^{\prime}}\left|\mathbf{p}, \mathbf{p}^{\prime}\right\rangle\right\rangle\left\langle\mathbf{p}, \mathbf{p}^{\prime}\right|=1
$$

We note that the eigenvalues of $L_{0}$ in Eq. (A10) are degenerated (e.g., for $\mathbf{p}^{\prime}=\mathbf{p}$ ) even though $\epsilon_{\mathbf{p}}$ are not degenerated. This is the intrinsic degeneracy of the Liouvillian mentioned in the Introduction.

In terms of the basis, we have a simple expression for a matrix element of an operator $A$ in the wave function space as

$$
A_{\mathbf{p}, \mathbf{p}^{\prime}} \equiv\left\langle\mathbf{p}|A| \mathbf{p}^{\prime}\right\rangle=\left\langle\left\langle\mathbf{p}, \mathbf{p}^{\prime} \mid A\right\rangle\right\rangle \text {. }
$$

A matrix element of the interaction Liouvillian is given by

$$
\begin{aligned}
& \left\langle\left\langle\mathbf{p}, \mathbf{p}^{\prime}\left|g L_{V}\right| \mathbf{p}^{\prime \prime}, \mathbf{p}^{\prime \prime \prime}\right\rangle\right\rangle \\
& \quad=\frac{1}{\hbar}\left[g V_{\mathbf{p}, \mathbf{p}^{\prime \prime}} \delta^{K r}\left(\mathbf{p}^{\prime}-\mathbf{p}^{\prime \prime \prime}\right)-\delta^{K r}\left(\mathbf{p}-\mathbf{p}^{\prime \prime}\right) g V_{\mathbf{p}^{\prime \prime \prime}, \mathbf{p}^{\prime}}\right] .
\end{aligned}
$$

We define the Wigner basis by

$$
\left.|\mathbf{k} ; \mathbf{P}\rangle\rangle \equiv\left|\mathbf{p}, \mathbf{p}^{\prime}\right\rangle\right\rangle
$$

with the relations in Eq. (10). The set of the Wigner basis also forms a complete orthonormal basis

$$
\begin{aligned}
\left\langle\left\langle\mathbf{k} ; \mathbf{P} \mid \mathbf{k}^{\prime} ; \mathbf{P}^{\prime}\right\rangle\right\rangle= & \delta^{K r}\left(k-k^{\prime}\right) \delta^{K r}\left(P-P^{\prime}\right) \\
& \times \prod_{j=1}^{N} \delta^{K r}\left(k_{j}-k_{j}^{\prime}\right) \delta^{K r}\left(P_{j}-P_{j}^{\prime}\right),
\end{aligned}
$$


and

$$
\left.\sum_{\mathbf{k}} \sum_{\mathbf{P}}|\mathbf{k} ; \mathbf{P}\rangle\right\rangle\langle\mathbf{k} ; \mathbf{P}|=1
$$

With the Fourier component of the interaction potential in Eq. (2), the Wigner representation of the matrix element of the interaction Liouvillian (A14) is given by

$$
\begin{aligned}
\langle\langle\mathbf{k} ; \mathbf{P}| & \left.g L_{V}\left|\mathbf{k}^{\prime} ; \mathbf{P}^{\prime}\right\rangle\right\rangle \\
= & \frac{1}{\Omega} \sum_{j=1}^{N} \frac{g V_{k-k^{\prime}}}{\hbar} \delta^{K r}\left(k-k^{\prime}+k_{j}-k_{j}^{\prime}\right) \prod_{i(\neq j)}^{N-1} \delta^{K r}\left(k_{i}-k_{i}^{\prime}\right) \\
& \times\left[\hat{\eta}_{P}^{\frac{\hbar}{2}\left(k-k^{\prime}\right)} \hat{\eta}_{P_{j}}^{-\frac{\hbar}{2}\left(k-k^{\prime}\right)}-\hat{\eta}_{P}^{-\frac{\hbar}{2}\left(k-k^{\prime}\right)} \hat{\eta}_{P_{j}}^{\frac{\hbar}{2}\left(k-k^{\prime}\right)}\right] \\
& \times \delta^{K r}\left(\mathbf{P}-\mathbf{P}^{\prime}\right)
\end{aligned}
$$

with $\hbar \mathbf{k}^{\prime} \equiv \mathbf{p}^{\prime \prime}-\mathbf{p}^{\prime \prime \prime}$ and $\mathbf{P}^{\prime} \equiv\left(\mathbf{p}^{\prime \prime}+\mathbf{p}^{\prime \prime \prime}\right) / 2$, where $\hat{\eta}_{P}^{p}$ and $\hat{\eta}_{P_{j}}^{p_{j}}$ are displacement operators acting on the momenta $P$ and $P_{j}$, respectively, as

$$
\hat{\eta}_{P}^{p} f(P)=f(P+p), \quad \hat{\eta}_{P_{j}}^{p_{j}} f\left(P_{j}\right)=f\left(P_{j}+p_{j}\right) .
$$

\section{APPENDIX B: TEMPERATURE DEPENDENCE}

In this appendix, we give an expression for the correction to the effective Liouvillian of the perfect Lorentz gas.

We expand the propagator in Eq. (30) as a power series of the ratio $m / M$,

$$
\begin{aligned}
& \frac{1}{z-(k-l) P / m-l P_{j} / M} \\
& =\sum_{n=0}^{\infty} \frac{\left(l P_{j} / m\right)^{n}}{[z-(k-l) P / m]^{n+1}}\left(\frac{m}{M}\right)^{n} .
\end{aligned}
$$

For each order term, one can perform the integration over momentum of $N$ heavy particles using the following formulas for the Gaussian integrals:

$$
\begin{aligned}
& \int_{-\infty}^{\infty} d P_{i}^{N} P_{j}^{2 n}\left(\frac{1}{2 \pi M k_{B} T}\right)^{N / 2} \prod_{i^{\prime}=1}^{N} \exp \left(-\frac{P_{i^{\prime}}^{2}}{2 M k_{B} T}\right) \\
& =I \times \int_{-\infty}^{\infty} d P_{j} P_{j}^{2 n}\left(\frac{1}{2 \pi M k_{B} T}\right)^{1 / 2} \exp \left(-\frac{P_{j}^{2}}{2 M k_{B} T}\right) \\
& =(2 n-1) ! !\left(\frac{M k_{B} T}{m}\right)^{n} m^{n},
\end{aligned}
$$

where $\int_{-\infty}^{\infty} d P_{i}^{N}$ stands for the integration over momenta of $N$ heavy particles, and

$$
\begin{aligned}
& \int_{-\infty}^{\infty} d P_{i}^{N} P_{j}^{2 n+1}\left(\frac{1}{2 \pi M k_{B} T}\right)^{N / 2} \prod_{i^{\prime}=1}^{N} \exp \left(-\frac{P_{i^{\prime}}^{2}}{2 M k_{B} T}\right) \\
& =I \times \int_{-\infty}^{\infty} d P_{j} P_{j}^{2 n+1}\left(\frac{1}{2 \pi M k_{B} T}\right)^{1 / 2} \exp \left(-\frac{P_{j}^{2}}{2 M k_{B} T}\right) \\
& =0
\end{aligned}
$$

with

$$
\begin{aligned}
I & \equiv \prod_{i(\neq j)}^{N} \int_{-\infty}^{\infty} d P_{i}\left(\frac{1}{2 \pi M k_{B} T}\right)^{1 / 2} \exp \left(-\frac{P_{i}^{2}}{2 M k_{B} T}\right) \\
& =1,
\end{aligned}
$$

which is a result of the normalization. Note that the $T$ dependence of Eq. (30) comes from even power in the series expansion with $(m / M)$ in Eq. (B1). Hence, we obtain

$$
\begin{gathered}
\int_{-\infty}^{\infty} d P_{i}^{N} \frac{\left(l P_{j} / m\right)^{2 n}}{[z-(k-l) P / m]^{2 n+1}}\left(\frac{m}{M}\right)^{2 n} \rho_{\mathrm{hev} .}^{\mathrm{eq}} \\
=\frac{(2 n-1) ! !}{[z-(k-l) P / m]^{2 n+1}}\left(\frac{l^{2} k_{B} T}{m}\right)^{n}\left(\frac{m}{M}\right)^{n},
\end{gathered}
$$

for the integrals in Eq. (30) over the momenta of the heavy particles. As a result, $T$ dependence appears already in the first order correction in $m / M$ in spite that Eq. (30) has a contribution in its even power. Then, we have Eq. (33).

\section{APPENDIX C: EXTENDED PSEUDO-EIGENSTATES}

In this appendix, we give a brief summary of the extended pseudo-eigenstate representation, which is divergent free near the singular points $k= \pm k_{P_{0}}$, introduced in [28].

First we introduce new eigenstates $\left.\left|\chi_{+; P_{0}}^{(k)}\right\rangle\right\rangle$ and $\left\langle\left\langle\tilde{\chi}_{-; P_{0}}^{(k)}\right|\right.$, which differ from $\left.\left|\phi_{-; P_{0}}^{(k)}\right\rangle\right\rangle$ and $\left\langle\left\langle\tilde{\phi}_{-; P_{0}}^{(k)}\right|\right.$ in their normalization, as

$$
\begin{aligned}
& \left|\chi_{+; P_{0}}^{(k)}\right\rangle \equiv\left[\frac{2\left(k^{2}-k_{P_{0}}\right)^{1 / 2}}{k}\right]^{1 / 2}\left|\phi_{+; P_{0}}^{(k)}\right\rangle, \\
& \left\langle\langle \tilde { \chi } _ { - ; P _ { 0 } } ^ { ( k ) } | \equiv i [ \frac { 2 ( k ^ { 2 } - k _ { P _ { 0 } } ) ^ { 1 / 2 } } { k } ] ^ { 1 / 2 } \left\langle\left\langle\tilde{\phi}_{-; P_{0}}^{(k)}\right|,\right.\right.
\end{aligned}
$$

where the factor $\left[k / 2\left(k^{2}-k_{P_{0}}^{2}\right)^{1 / 2}\right]^{1 / 2}$ is the divergent factor in the coefficients of the eigenvectors (45). Since the divergent factor is removed in the new the eigenstates $\left.\left|\chi_{+; P_{0}}^{(k)}\right\rangle\right\rangle$ and $\left\langle\left\langle\tilde{\chi}_{-; P_{0}}^{(k)}\right|\right.$, they do not diverge at the points $k= \pm k_{P_{0}}$. The pseudo-eigenstates that form a complete orthonormal basis set with $\left|\chi_{+; P_{0}}^{(k)}\right\rangle$ and $\left\langle\left\langle\tilde{\chi}_{-; P_{0}}^{(k)}\right|\right.$, denoted by $\left.\left.\mid \chi_{-; P_{0}}^{\prime(k)}\right\rangle\right\rangle$ and $\left\langle\left\langle\tilde{\chi}_{+; P_{0}}^{\prime(k)}\right|\right.$, are defined through the following relations:

$$
\begin{aligned}
{\left[\psi_{B}^{(k)}-z_{-; P_{0}}^{(k) B} \hat{I}_{2}\right]\left|\chi_{-; P_{0}}^{\prime(k)}\right\rangle=} & \left.=\frac{g^{2} \gamma_{P_{0}}}{2}\left|\chi_{+; P_{0}}^{(k)}\right\rangle\right\rangle, \\
\left\langle\left\langle\tilde{\chi}_{+; P_{0}}^{\prime(k)}\right|\left[\psi_{B}^{(k)}-z_{+; P_{0}}^{(k)} \hat{I}_{N}\right]\right. & =\frac{g^{2} \gamma_{P_{0}}}{2} \mid\left\langle\tilde{\chi}_{-; P_{0}}^{(k)}\right| .
\end{aligned}
$$

We impose the following normalization conditions for the right- and left-extended pseudo-eigenstates:

$$
\left\langle\left\langle\tilde{\chi}_{+; P_{0}}^{\prime(k)} \mid \chi_{+; P_{0}}^{(k)}\right\rangle\right\rangle=1, \quad\left\langle\left\langle\tilde{\chi}_{-; P_{0}}^{(k)} \mid \chi_{-; P_{0}}^{\prime(k)}\right\rangle=1 .\right.
$$

Then, we have right- and left-pseudo-eigenstates,

$$
\begin{aligned}
& \left.\left.\left|\chi_{-; P_{0}}^{\prime(k)}\right\rangle=a_{-; P_{0}}^{\prime(k)}\left|k ; P_{0}\right\rangle\right\rangle+b_{-; P_{0}}^{\prime(k)}\left|k ;-P_{0}\right\rangle\right\rangle, \\
& \left\langle\left\langle\tilde{\chi}_{+; P_{0}}^{\prime(k)}\right|=a_{+; P_{0}}^{\prime(k)}\left\langle\left\langle k ; P_{0}\right|+b_{+; P_{0}}^{\prime(k)}\left\langle\left\langle k ;-P_{0}\right|\right.\right.\right.
\end{aligned}
$$


with

$$
\begin{aligned}
& a_{ \pm ; P_{0}}^{(k)}=\frac{1}{2}\left[1 \pm \frac{\left(k^{2}-k_{P_{0}}^{2}\right)^{1 / 2}}{k}\right]^{1 / 2}, \\
& b_{ \pm ; P_{0}}^{\prime(k)}=-\frac{i}{2}\left[1 \mp \frac{\left(k^{2}-k_{P_{0}}^{2}\right)^{1 / 2}}{k}\right]^{1 / 2} .
\end{aligned}
$$

The vectors $\left.\left\{\left|\chi_{+; P_{0}}^{(k)}\right\rangle\right\rangle,\left|\chi_{-; P_{0}}^{\prime(k)}\right\rangle\right\rangle,\left\langle\left\langle\tilde{\chi}_{+; P_{0}}^{\prime(k)}\right|,\left\langle\left\langle\tilde{\chi}_{-; P_{0}}^{(k)}\right|\right\}\right.$ satisfy the orthogonality

$$
\left\langle\left\langle\tilde{\chi}_{+; P_{0}}^{\prime(k)} \mid \chi_{-; P_{0}}^{\prime(k)}\right\rangle\right\rangle=0, \quad\left\langle\left\langle\tilde{\chi}_{-; P_{0}}^{(k)} \mid \chi_{+; P_{0}}^{(k)}\right\rangle\right\rangle=0,
$$

and completeness

$$
\sum_{P_{0}}\left(\left|\chi_{+; P_{0}}^{(k)}\right\rangle\right\rangle\left\langle\left\langle\tilde{\chi}_{+; P_{0}}^{\prime(k)}|+| \chi_{-; P_{0}}^{\prime(k)}\right\rangle\right\rangle\left\langle\left\langle\tilde{\chi}_{-; P_{0}}^{(k)}\right|\right)=\hat{p}^{(k)},
$$

with

$$
\left.\hat{p}^{(k)}=\sum_{P}|k ; P\rangle\right\rangle\langle k ; P|
$$

[1] L. Van Hove, Physica 23, 441 (1957).

[2] S. Nakajima, Prog. Theor. Phys. 20, 948 (1958).

[3] R. Zwanzig, J. Chem. Phys. 33, 1338 (1960).

[4] P. Resibois, Physica 27, 541 (1961).

[5] I. Prigogine, Nonequilibrium Statistical Mechanics (John Wiley \& Sons Inc., New York, 1962).

[6] R. Zwanzig, Physica 30, 1109 (1964).

[7] N. N. Bogoliubov, Problems of a Dynamical Theory in Statistical Physics (Gostekhizdat, Moscow, 1946) (in Russian); in Studies in Statistical Mechanics, vol. 1, edited by J. de Boer and G. E. Uhlenbeck (North-Holland Pub., Co., Amsterdam, 1962), p. 5 (English translation).

[8] M. S. Green, J. Chem. Phys. 25, 836 (1956).

[9] E. G. D. Cohen, Physica 28, 1025 (1956).

[10] R. Zwanzig, Phys. Rev. 124, 983 (1961).

[11] H. B. Hollinger, J. Chem. Phys. 36, 3208 (1962).

[12] R. Balescu, Equilibrium and Nonequilibrium Statistical Mechanics (John Wiley \& Sons, Inc., New York, 1975).

[13] L. D. Landau and E. M. Lifshits, Statistical Physics, 3rd ed., Part 1 (Butterworth-Heinemann, Oxford, 1980).

[14] T. Petrosky and I. Prigogine, Chaos, Solitons \& Fractals 7, 441 (1996).

[15] T. Petrosky and I. Prigogine, in Advances in Chemical Physics, vol. 99, edited by I. Prigogine and Stuart A. Rice (John Wiley \& Sons Inc., New York, 1997), Chap. 1, p. 1.

[16] T. Petrosky, Prog. Theor. Phys. 123, 395 (2010).

[17] H. Feshbach, Ann. Phys. (NY) 5, 357 (1958).

[18] H. Feshbach, Ann. Phys. (NY) 19, 287 (1972).
[19] T. Petrosky, I. Prigogine, and S. Tasaki, Physica A 173, 175 (1991).

[20] N. Hatano, Fortschr Phys. 61, 238 (2013).

[21] S. Tanaka, K. Kanki, and T. Petrosky, Phys. Rev. B 80, 094304 (2009).

[22] L. Boltzmann, Further studies on the thermal equilibrium of gas molecules (Sitzungsberichte der kaiserlichen Akademie der Wissenschaften, Vienna, 1872) (in German); Stephen G. Brush, in History of Modern Science 1, The Kinetic Theory of Gases: An Anthology of Classical Papers with Historical Commentary, edited by Nancy S. Hall (Imperial College Press, London, 2003), p. 262 (English translation).

[23] R. Loudon, The Quantum Theory of Light, 3rd ed. (Oxford University Press, Inc., New York, 2001).

[24] T. Petrosky, Foundations Phys. 29, 1417 (1999).

[25] T. Petrosky, Foundations Phys. 29, 1581 (1999).

[26] T. Petrosky, in Advances in Chemical Physics, Vol. 122, edited by Ioannis Antoniou (John Weily \& Sons, Inc., New York, 2002), Chap. 8, p. 129.

[27] Z. L. Zhang, Irreversibility and extended formulation of classical and quantum nonintegrable dynamics, Ph.D. thesis, The University of Texas at Austin, 1995.

[28] K. Hashimoto, K. Kanki, H. Hayakawa, and T. Petrosky, Prog. Theor. Exp. Phys. 2015, 023A02 (2015).

[29] R. Balescu, Statistical Mechanics of Charged Particles (John Wiley \& Sons Inc., New York, 1963).

[30] A. Messiah, Quantum Mechanics (North-Holland Pub., Co., Amsterdam, 1961).

[31] P. Resibois and M. De Leneer, Classical Kinetic Theory of Fluids (John Wiley \& Sons Inc., New York, 1977). 